\title{
Analysis of Technology Evolution Trends for Predicting Future Technologies
}

\author{
Yong-Won Song1)
}

\begin{abstract}
Establishing a method for the prediction of future technology is crucial in a modern society in which technology develops rapidly and the lifetime of products is very short. However, many future forecasting methods, such as the Delphi technique, scenario planning, and futures wheel, have the potential to produce false results because they rely on individual or collective intelligence in subjective ways[1]. Therefore, the development of an objective method is necessary. G. Altshuller, the founder of the theory of inventive problem solving, showed that systems develop according to objective laws, and these laws can be used for solving technical problems. The technology evolution laws he discovered not only could be used to solve technical problems but also opened up new ways to predict future technologies. This paper shows 11 trends of technical system evolution existing in the history of technology development and how these trends can be used to predict future technologies. This new forecasting method based on evolutionary patterns is intended to help in predicting future technologies objectively, as opposed to the subjective views of analysts.
\end{abstract}

Keywords: Trend, TRIZ, Evolution, Technical System, S-curve, Predict

\section{Introduction}

The ability to establish appropriate strategies by understanding and predicting technology development is important for researchers and leaders in the rapidly changing era of the fourth industrial revolution. The Delphi technique, a representative method for predicting future technologies, involves establishing future strategies by surveying numerous experts, analyzing their opinions, and reaching an agreement while correcting errors through discussion. Specifically, it involves organizing a group of experts, collecting their opinions by asking them about their outlook for the future, and making the final predictions by discussing and reconciling the opinions multiple times to establish future strategies[2]. A technology roadmap can also be prepared and used through patent analysis. Most of the top global companies develop their

Received(May 15, 2020), Review Result(1st: July 3, 2020, 2nd: August 19, 2020), Accepted(September 25, 2020)

1) (Professor) 15073 Dept. Nano \& Semiconductor Engineering, Korea Polytechnic Univ., Sangidaehak-ro, Siheung-si, Gyeonggi-do, Korea

email: ywsong@kpu.ac.kr 
own analysis methods, prepare technical forecasts, and use them to establish internal $R \& D$ strategies. For example, IBM has been identifying global trends and topics every year since 1982 using patent and technical data, and they establish and implement market-leading R\&D strategies using global jamming (online or offline brainstorming), in which company experts, academics, entrepreneurs, and even ordinary individuals participate. However, most of these existing methods require considerable time and cost. Conglomerates or government agencies with sufficient time and economic margins use such methods to establish future technology development strategies. It is difficult for ordinary researchers or small- and medium-sized companies with limited time and economic resources to use these existing methods. In addition, such methods have frequently led to wrong decisions because they rely on the subjectivity of individual experts. Therefore, it is necessary to develop a new methodology for establishing new future technology development strategies that is objective and can easily be utilized by general researchers.

Technical system evolution trends can be analyzed and utilized to develop a method for establishing future technology development strategies in an objective manner. For example, it is possible to objectively predict the direction of technologies to be developed and establish strategies using Moore's law, which states that the amount of data that can be stored in a microchip doubles every 18 months. Moore's law in the semiconductor sector shows the possibility of developing a methodology for predicting the future relatively easily and objectively through the analysis of technology evolution trends that exist in the technical system development process.

G. Altshuller, a Russian scientist, discovered through patent analysis that technical systems evolved according to objective patterns and called those patterns the laws of technical system evolution. He also discovered that some principles were used repeatedly for solving complex problems and called them inventive principles. He developed the theory of inventive problem solving (TRIZ) based on the laws of technical system evolution and the inventive principles[3]. With advances in TRIZ, B. Zlotin utilized technology evolution trends to create a methodology for deriving future technology development strategies[4]. Technology evolution trends can be used to predict the future without being affected by the subjectivity of individual researchers because they are objective laws for the development of technical systems such as Moore's law. If the technology evolution trends in all industrial technology sectors are analyzed instead of those in specific sectors, such as semiconductors, it is possible to develop an innovative methodology for future strategies. However, further research is required on the laws of technical system evolution because they do not include recent trends in the latest industries such as IT, 
artificial intelligence, robots, and big data that lead the fourth industrial revolution as they were developed mainly based on patents in the fields of machinery, materials, shipbuilding, and aviation in Russia from the 1950s to 1980s[5].

This study summarized the trends in technical system evolution studied by G. Altshuller until the 1980s in the traditional industrial sectors. Futhermore, it analyzed the development trends of modern technical systems, which are leading the fourth industrial revolution. An attempt was made to develop a new methodology for predicting the future technologies of technical systems which is not influenced by the subjectivity of individual researchers by identifying comprehensive trends in the technical system evolution.

\section{Trends in Technical System Evolution}

\subsection{Birth, Growth, Maturity, and Decline of Technical Systems}

Humans create systems (products or organizations) to perform desired functions. Systems are continuously created and developed owing to the endless growth and diversification of human needs. Ideality can be used as a qualitative index for evaluating the development of systems[6].

$$
\text { Ideality }=\frac{\sum \text { Useful Functions }}{\sum(\text { Cost }+ \text { Harm ful Functions })}
$$

Ideality (1) is obtained by dividing the sum of the useful functions of an observed system by the sum of its manufacturing or operating cost and harmful functions. In other words, the development of a system means an increase in ideality because useful functions are improved or increased while cost and harmful actions (functions) are decreased. Functions can be evaluated using various parameters, and the one that can express a representative feature of a system is defined as the main parameter of value (MPV). For example, it can be said that the MPV of an airplane is its speed and that of a computer storage medium is the number of bits that can be stored in a unit area.

Considering the development of flight vehicles, the first to realize the human need to fly were the Montgolfier brothers[7]. They came up with the idea when they saw laundry that was drying over a fire floating. They created a balloon with cloth and paper and set a fire underneath it to allow the hot air to move into the balloon. The first hot air balloon was quickly torn and destroyed; however, the time it remained aloft could be gradually increased by improving the material. For the first-generation hot air balloons, the MPV was the distance 
traveled. (The floating time was only important at the beginning. The ability to fly longer and farther, however, became an important core value, and for the flight vehicles developed later, MPV was the speed). The first hot air balloon made of paper and pieces of cloth in December 1782 was destroyed after a flight of few dozen of meters; however, the 79-m3 hot air balloon that was made of hemp cloth in June the following year flew $2 \mathrm{~km}$ and rose to a height of $180 \mathrm{~m}$. With the spread of this principle, many people developed hot air balloons, dramatically increasing the distance traveled. However, as the size of the balloons could not be increased infinitely and it was difficult to float large balloons, the increase in the MPV was slowed down and saturated, resulting in a constant MPV. A representation of the MPV change over time is referred to as an S-curve.

When the MPV of a system reaches its limit, researchers seek a new principle that can serve the same function. Instead of filling a balloon with hot air, researchers came up with the idea of filling a hot air balloon with light hydrogen gas. In 1852, Henri Jacques Giffard, a French inventor, created an airship filled with hydrogen gas that travelled using propeller rotation caused by a steam engine and succeeded in flying at a speed of $10 \mathrm{~km} / \mathrm{h}$, thereby opening a new generation of flight vehicles. However, Giffard's airship also reached a time of maturity when the MPV no longer rose after reaching an S-curve top of its own, paving the way for the Wright brothers' glider that significantly improved the speed of an airplane. Aircraft with jet engines followed, and they evolved into flight vehicles with ultrasonic speed. [Fig. 1] shows the development history of flight vehicles.

\section{S-Curve of flight vehicles}

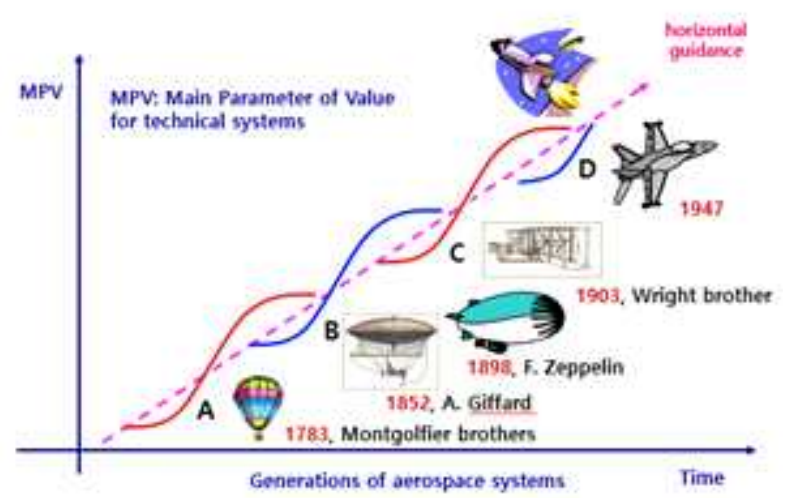

[Fig. 1] S-curve of Technical System Evolution for Flight Vehicles

As shown in [Fig. 1], flight vehicle technologies created by one principle develop in the shape of an S-curve and become obsolete when the MPV reaches its limit, and they are 
replaced with a system operating on a new principle. Through the development process with cycles of "birth-growth-maturity-decline," systems that implement the functions desired by humans evolve into new generations, and the MPV continues to grow. G. Altshuller, who created TRIZ, analyzed the development process of patents and many technical systems and argued that "technical systems develop according to objective laws. These laws can be perceived and used."[3] He analyzed trends in technical system evolution and used them as tools for inventive problem solving[8]. Evolution trends can be used to solve problems, predict future technologies, and establish development strategies. In this study, the nine technology evolution trends analyzed by Altshuller are summarized, and an attempt is made to analyze new trends that can be seen in the recent history of technology development.

\subsection{Trends in Technical System Evolution}

\subsubsection{Trend of the System Completeness of the Parts of the System}

A technical system consists of many components; however, four core components can be analyzed as follows.

(1) Working unit - A core element that performs the function of a technical system to the operating target. For example, in the case of an electric fan, the working unit is the blades that generate the wind by moving air, i.e., the operating target.

(2) Engine - A technical system component that generates the power required for the working unit to perform its function. The main function of the engine is to convert the energy supplied from the outside into the form of energy required by the working unit. Therefore, the engine can be referred to as an energy conversion device. In an electric fan, the engine is the electric motor, which converts the electric energy supplied from the outside into the mechanical rotational energy required for the system to perform its function.

(3) Transmission - A system component that transmits the power generated by the engine to the working unit. In the case of an electric fan, the transmission is the parts that transmit power from the motor to the blades.

(4) Control unit - A system component that controls the technical system to perform its function in a way desired by humans. In the case of an electric fan, the control unit is the buttons for adjusting the intensity of the wind and making the fan rotate or operating the timer.

A technical system can be divided into the working unit, engine, transmission, and control 
unit. It performs its function while interacting with the surrounding energy source, operating target, and controlling entity. [Fig. 2] shows the schematic diagram of a technical system.

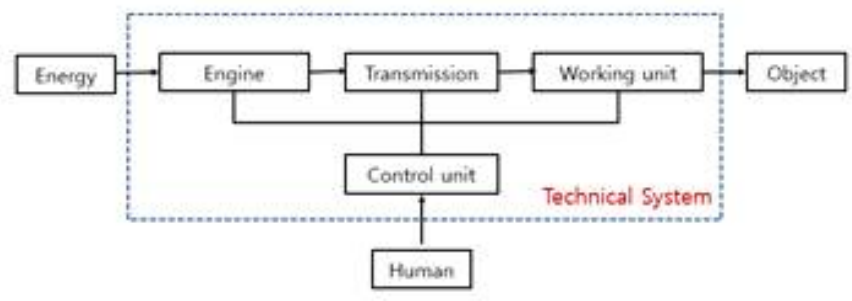

[Fig. 2] Components of a Technical System

In the history of technical system development, the working unit that directly performs a function was created first followed by the development of the transmission, engine, and control unit, thereby leading to a complete system with four parts. In the history of electric fans, fans with only a working unit were used at the beginning. Humans played the roles of the engine, transmission, and control unit. With the development of technology, such fans developed into primitive automatic fans, which used a heavy weight (engine) and pulley and were operated by a string (transmission) connected to the weight as it dropped. They developed into fans in which the blades were rotated by winding clockwork and culminated in the birth of today's electric fans.

\subsubsection{Trend of the Energy Conductivity of the System}

For the efficient operation of a technical system, the energy generated by the engine must flow well into the working unit through the transmission. The trend of energy conductivity of the system represents the technical system development in a direction that allows the efficient transmission of energy. For high energy conductivity, it is necessary to minimize changes in energy behavior and shorten the path from the engine to the working unit. In the case of automotive internal combustion engines, fuel is converted into thermal energy through combustion, and the expansion force of the thermal energy is used to move the pistons and thereby to generate mechanical power. When the energy is converted several times in the form of "chemical energy $\rightarrow$ thermal energy $\rightarrow$ piston reciprocating energy $\rightarrow$ rotational energy using cranks," significant losses occur. On the other hand, if an electric motor is used as the engine, the conversion process is reduced by one step in the form of "electric energy $\rightarrow$ rotational energy," resulting in the evolution into a much more efficient system. Moreover, the direct connection of the motor, i.e., the power engine, to the wheels will develop an even more 
efficient system due to the shorter energy transmission path.

\subsubsection{Trend of the Harmonizing Rhythms of the Parts of the System}

Systems develop so that there will be a good harmony among the technical system components or surrounding elements in terms of shape, material, color, and rhythm. In the case of bolts and nuts, the "unified" and "Whitworth" types are currently used. The thread angle of the unified screw thread is $60^{\circ}$ and that of the Whitworth screw thread is $55^{\circ}$. Bolts and nuts are manufactured and used according to these thread angles. In the case of bookcases, partitions were made in a specific size in the past; however, they are now made to conveniently store various books considering the sizes of the books published. The steering wheel of a good car helps the driver steer the car comfortably through a good harmony with the hands.

When selecting system components, many elements need to be considered for a rhythm harmony in terms of materials. If two elements in contact have different thermal expansion coefficients, wear or fracture may occur between the two elements in a temperature-changing environment. If two materials have significantly different standard reduction potentials, corrosion occurs. Therefore, it is necessary to consider mechanical, electrical, magnetic, optical, thermal, and corrosive properties for a good harmony when selecting the materials of adjacent elements.

\subsubsection{Trend of Increasing the Degree of Ideality of the System}

The ideality index described as equation (1) above can be used to evaluate technical systems. Technical systems develop in a direction that increases functions compared to the cost (price). Cost is a concept that includes the weight, volume, energy consumption, and harmful phenomena of the system as well as its price. The trend of increasing the degree of ideality of the system can be said to be a core trend that explains the first vector direction for system development. The following several aspects can be considered for the application of this trend: (1) what are the functions of the system, (2) what are the harmful actions (functions) of the system, and (3) what functions are good if added to the system?

The world's first cellphone was developed by a research team at Motorola led by Dr. Martin Cooper in 1973[9]. The phone weighted approximately $850 \mathrm{~g}$. The DynaTAC 8000X, a phone commercialized in 1983, had a large size of $330 \times 44.4 \times 88.8 \mathrm{~mm}$. It could provide an approximately 30-min conversation with a 4-h continuous standby when charged for $8 \mathrm{~h}$, and its price was \$3,995[10]. Today's smartphones enable a continuous standby longer than $30 \mathrm{~h}$ even though they are lighter and smaller due to the significant increase in ideality, and they 
have countless functions and low prices.

\subsubsection{Trend of Increasing Dynamism}

Technical systems evolve toward dynamic movement so that functions can be performed more efficiently. They become changeable and adapt quickly to environmental conditions. For example, early airplanes had fixed wheels for takeoff and landing. As the wheels were always exposed to the outside environment, they created air resistance during flight, thereby reducing the flight efficiency. With advances in technology, the wheels were designed to move dynamically, and they developed into dynamic systems that can be folded into the airplane after take-off.

\subsubsection{Trend of Increasing the Substance-field Involvement}

The functions of a technical system are performed when the required force (energy) is applied to the elements of the system. The relationship between the material and energy that realizes such functions can be explained using the "substance-field interaction"[11]. Experts in TRIZ classify these fields into mechanical, acoustic, thermal, chemical, electrical, magnetic, and electromagnetic from an engineering perspective for the implementation of functions [Table 1].

[Table 1] Types and Components of the Engineering Fields

\begin{tabular}{|c|c|}
\hline Fields & Types \\
\hline \multirow{5}{*}{ Mechanical } & Gravity, collision, friction (force), and direct contact \\
\hline & Vibration, resonance, impact, and waves \\
\hline & $\begin{array}{l}\text { Gas/fluid dynamics), wind (wind power), } \\
\text { compression, and vacuum }\end{array}$ \\
\hline & Mechanical action (e.g. machining and drilling) \\
\hline & Deformation, mixing, additives (for mechanical action), and explosion \\
\hline Acoustic & $\begin{array}{l}\text { Voice (sound: 20-15,000 Hz), ultrasound, ultra-low frequencies (inaudible } \\
\text { sound), cavitation }\end{array}$ \\
\hline \multirow{3}{*}{ Thermal } & Heating, cooling, insulation, and thermal expansion \\
\hline & Phase/state change, exothermic reaction, and endothermic reaction \\
\hline & Fire, heat radiation, and convection \\
\hline \multirow{4}{*}{ Chemical } & Chemical reaction, reactants, chemicals, and compounds \\
\hline & Catalysts, inhibitors, and indicators $(\mathrm{pH})$ \\
\hline & Dissolution, crystallization, and polymerization \\
\hline & Odor, taste, color change, and $\mathrm{pH}$ \\
\hline \multirow{4}{*}{ Electrical } & Electrostatic charges, conductors, and non-conductors \\
\hline & Electric fields and current \\
\hline & Superconductivity, electrolysis, and piezoelectric phenomenon (effect) \\
\hline & Ionization, discharge, and spark \\
\hline Magnetic & Magnetic fields, magnetic materials, and magnetic induction \\
\hline Electromag & Electromagnetic waves (X-ray, Microwaves, etc.) \\
\hline
\end{tabular}


As for systems that perform the function of cutting materials, mechanical saws were developed and used; subsequently, other cutting tools that use acoustic fields, such as ultrasound and thermal fields, or chemicals, such as hydrofluoric acid (HF), were developed. It is also possible to develop cutting machines that use electrical fields or equipment that uses a laser (electromagnetic field). Systems that perform certain functions evolve from the systems that use mechanical fields into those that use electrical/magnetic fields or electromagnetic fields in the development process.

To improve system performance, systems that are implemented using one field develop into complex 'substance-field' systems with two or three fields. For example, washing machines that removed impurities by applying mechanical motion evolved into complex substance-field systems that increase the water temperature or add detergent to improve the washing function.

\subsubsection{Trend of Non-uniform Development of System Elements}

A system consists of multiple parts (elements). For the development of a system, efforts are made to meet the requirements of some elements rather than harmoniously improving all the parts. This causes conflict among the system components. This trend can be examined with the example of bicycle development process as follows. Early bicycles did not have pedals, and the kicking of the feet was required to move them. As this caused slow speed and inconvenience, efforts were made to improve the speed of bicycles. The development of the technology to mount pedals at the front wheel in 1840 increased the speed of bicycles[12]. However, as the speed increased, accidents began to occur, as the bicycles could not be stopped. Thus although the function of running well was important, a mounted brake system as a device for stopping bicycles was required. After the development of the brake system, research was conducted into achieving higher speeds, leading to the development of a chain-drive system in 1884, which could dramatically increase the bicycle speed through its connection to the rear wheel. The developed system, however, caused the inconvenience of constantly moving the feet with the pedals. To improve this, the overrun (free rotation) clutch was invented in 1897, and it developed into modern bicycles in which the wheels move without having to move the pedals after reaching the desired speed.

The development process of technical systems frequently shows that an improvement in one subsystem leads to a degradation in another subsystem. This is defined as a "contradiction" problem in TRIZ, and 40 invention principles to address this problem were developed for the 
creative development of systems[13].

\subsubsection{Trend of Transition from Macro to Micro Levels}

Making the working unit of a technical system with a bi-system, tri-system, or multi-system rather than a mono-system leads to an advanced system that performs its function more efficiently. When the evolution of propellers is considered in the airplane development process, single-blade propellers have a simple structure but cannot produce a large thrust. Therefore, they are not used except for lightweight unmanned aircraft. More advanced double propellers are used in light aircraft due to the balance of two blades and improved thrust. Airplanes with more advanced multiple propellers can carry heavy cargo.

In the course of the development of materials for the working unit at micro levels, these materials have evolved from a bulk form to a powder form and from a powder form to a liquid/gas form, eventually leading to an evolution in the forms of fields, such as electrical, magnetic, and electromagnetic fields. For example, cutting machines developed from bulk forms, such as saws, to water jet cutters that use high-pressure water. More advanced devices are plasma cutters and cutters that use a laser (electromagnetic field). The history of printer development shows that the early bulk-type working unit in the form of a typewriter was replaced with inkjet printers that use liquid ink, and laser printers that use a laser have been developed and used of late.

\subsubsection{Trend of Transition (Integration) to a Super-system}

Technical systems develop individually to efficiently perform the given functions and then evolve in the direction of being combined with super-level systems that perform similar or opposite functions. The functions of cameras and MP3 players were integrated into smartphones. Kitchen tools currently available also reflect the integration trend. Electric heaters for cooking foods that apply heat and induction coils using electromagnetic waves have their own benefits and shortcomings. Induction coils have a high energy efficiency through fast heating and reduced cooking time; however, they require metal containers and destroy some nutrients during cooking. Electric heaters, on the other hand, can maintain the unique taste of food and protect nutrients, but they require relatively longer cooking times and have a low energy efficiency. Integrated cookers that have the benefits of both systems have been developed and are available in the market. Integrated generators that are less affected by the weather have been manufactured and utilized by integrating solar panels with wind power generators. 
The above nine trends in the technical system evolution were classified by Altshuller and his students by analyzing patents, papers, and products until 1985. Since then, many advances have been made in modern science and technology in the fourth industrial revolution following the internet-based information age, and new trends in technology development have emerged.

\subsubsection{Trend of Increasing Connectivity}

Systems develop independently and then evolve by forming a network through "connections" with other nearby systems. They are organically linked with other systems and are able to exchange information through the network, and they evolve into a stage in which the functions desired by humans can be intelligently performed through the combination with software technology. Connectivity is evolving from the people-to-people connection that used the internet to hyper-connectivity systems in which people-to-object and object-to-object connections, i.e., everything-to-everything connections, occur. With sensors attached to objects and access to the wireless internet, new functions desired by humans are performed. The sensors attached to a refrigerator detect insufficient food items, and they can send the information to a smartphone or place an order to a nearby store. Various sensors attached to a smart watch check the health status of a person, and they can send the information to a smart phone or contact a hospital or 911 in case of an emergency to save the patient who is in danger.

Automobiles independently performed the transportation function in the past, but they are developing into "connected cars" that perform various functions through connections with surrounding objects and the internet. Vehicle-to-infra (V2I) communication technology that allows highway tolls to be paid at toll booths using GPS and a terminal has been used. Connectivity is further developing into vehicle-to-vehicle (V2V) communication technology that allows vehicles to exchange information as they are connected to each other. As vehicles exchange information with other nearby vehicles in real time through the developed V2V technology, they can avoid congestion and perform safe driving by identifying the road traffic status.

\subsubsection{Trend of Decreasing Human Involvement}

A technical system is divided into the engine, transmission, working unit, and control unit. Humans control a technical system through the control unit. With the development of systems, the control unit moves toward easier control by humans. It gradually evolves into a smart system that minimizes human involvement and performs the functions desired by humans by itself. For example, early steering systems for automobiles were mechanical and required 
considerable force to rotate the steering wheel. Later, electronic steering systems were developed, making it possible to rotate the steering wheel with little force. Early manual transmissions that required gear shifting by the driver according to the speed developed into automatic transmissions, enabling convenient driving. Technology does not stop here and develops into autonomous vehicles that minimize driver involvement and perform control by themselves. Building entrances or office doors were manually opened or closed by human hands, but they are changing so that they can be opened and closed automatically using sensors when a person approaches.

The development of artificial intelligence (AI) technology, which learns the ways people judge and make decisions with information collected through sensors, data mining, and big data technology, is breaking new ground for technical system evolution. Advances in AI technology will be applied to most technical systems, causing them to develop into smart systems that can automatically perform the functions desired by humans without human involvement in the future.

The nine evolution trends analyzed by the experts in TRIZ until 1985 were summarized along with two additional trends that were discovered later in the industrial technology development process. The nine evolution trends were as follows: the "trend of the system completeness of the parts of the system," "trend of energy conductivity of the system," "trend of harmonizing rhythms of parts of the system," "trend of increasing the level of ideality of the system," "trend of increasing dynamism," "trend of increasing the substance-field involvement," "trend of non-uniform development of system elements," "trend of transition from macro- to micro-levels," and "trend of transition (integration) to a super-system." The two newly analyzed trends in the history of modern technology development that led the fourth industrial revolution were the "trend of increasing connectivity" and "trend of decreasing human involvement."

\section{Conclusion}

Research on technical system evolution patterns was conducted to predict future technologies in an objective manner. Technology evolution trends can be used as objective indices for predicting the future development directions of current products or technologies. First, the target system was analyzed in terms of the 11 evolution trends to identify its current positions. When the current positions compared to the highest levels of the technology evolution trends 
were identified, it is possible to identify areas that made considerable development and areas that require further development in terms of each trend. Based on this, development strategies can be established for improving the areas that require further development [Fig. 3].

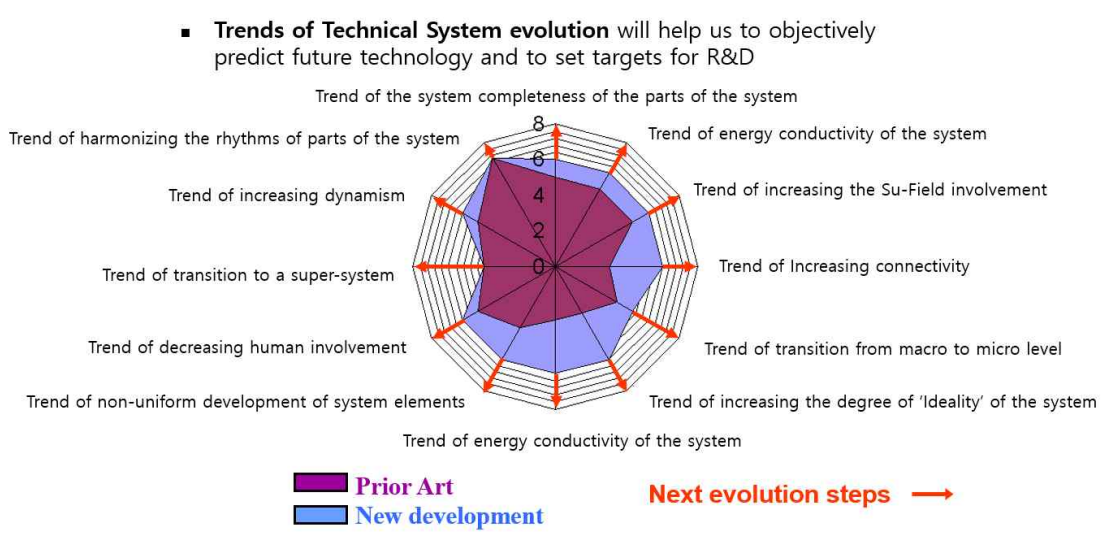

[Fig. 3] Future Prediction using Evolution Trends

There is, however, another important element to be considered for predicting future technologies. These are the macro-trends in the society, culture, economy, and politics of the people who select and use technical systems. It is necessary to develop a methodology for predicting future technologies by combining technology evolution trends with macro-trends.

\section{Acknowledgement}

This research was supported by Basic Science Research Program through the National Research Foundation of Korea (NRF) funded by the Ministry of Education (2018R1D1A1B07049244)

\section{References}

[1] Kycharavy, D. and De Guio, R. Problems of Forecasting. Proceedings of the $5^{\text {th }}$ ETRIA TRIZ Future Conference, ISBN 3-7011-0057-8, (2005), November 16-18; Graz, Austria

[2] International Future Society, The Strategic Methodology for Future Studies, Korea: Donam, (2014)

[3] G. S. Altshuller, Creativity as an Exact Science, CRC Press, (1984)

[4] https://triz-journal.com/, Jan 05 (2001) 
[5] Genrich Altshuller, Lev Shulyak, And Suddenly the Inventor Appeared: TRIZ, the Theory of Inventive Problem Solving, Growth Opportunity Alliance of Lawrence, (2004)

[6] Karen Gadd, TRIZ for Engineers: Enabling Inventive Problem Solving, USA: A John Wiley \& Sons, Ltd., Publication, (2011)

[7] https://en.wikipedia.org/wiki/Montgolfier_brothers, Wikipedia, Sept 13 (2020)

[8] Bukhman, I., TRIZ: Technology for Innovation, GS Intervision, (2014)

[9] https://ko.wikipedia.org/wiki/\%ED\%9C\%B4\%EB\%8C\%80_\%EC\%A0\%84\%ED\%99\%94, Sept 13 (2020)

[10] https://blog.uplus.co.kr/2141, Sept 13 (2020)

[11] Y.W. Song, K.M, Kim and S.H. Kim, Substance-Field Analysis and Standards, The theory of inventive problem solving, TRIZ, Korea Standard Association Media, (2018)

[12] V. Fey, E. Rivin, Innovation on demand: New product development using TRIZ, Cambridge University Press, (2010)

[13] S. Ikovenko, M. Przymusiala, S. Yatsunenko, M. Barkan, P. Karendal, S. Kobyakov, J. Obojski, Z. Vintman, Theory of Inventive Problem Solving - State of the Art, Poland: Novismo, (2019) 